\title{
Beiträge zur histologischen Kenntnis des Nervus trigeminus.
}

\author{
III. Mitteilung. \\ Über die multipolaren Zellen im Ganglion semilunare. \\ Von \\ Dr. med. Genichiro Takeda. \\ (Aus dem Anatomischen Institut der Kaiserlichen Universität Kyoto, Japan)
}

Mit 1 Abbildung im Text.

Die Nervenzellen der Zerebrospinalganglien werden besonders nach Anzahl, Gestalt und Verlanf ihrer Fortsätze in verschiedene 'Typen eingeteilt. Nur sehr selten stösst man auf die interessanten Multipolarzellen. Über das Schicksal und den Charakter ihrer Fortsätze ist man noch nicht völlig im klaren. Doch scheint es mir ihrer Natur nach, dass sie im allgemeinen als dendritenartige Ausläufer aufzufassen sind.

Dass sich in den Spinalganglien neben den typischen Unipolarzellen und verhältnismässig seltenen bipolaren Zellen und Übergangsformen auch einige multipolare Nervenzellen sporadisch vorfinden, wissen wir seit dem Jahre 1893, als Disse (3) der siebenten Anatomenverammlung zu Göttingen Präparate von Froschlarven vorlegte. Er untersuchte bei Froschlarven in den jüngsten Stadien, bei denen die hinteren Extremitäten noch nicht entwickelt waren, die Zellen der Spinalganglien auf die Zahl ihrer Fortsätze und auf das Vorkommen von Dendriten und bediente sich dabei des Verfahrens von Ramón y Cajal (mehrtägige Härtung in 3-proc. Natr. b:chrom. mit Osmiumzusatz, dann Einlegen in eine 3/4proc. Lösung von Argent. nitr.). Es liessen sich 2 Arten von Zellen nachweisen; die eine Zellart hatte ausser den beiden Nervenfortö̈tzen noch einen dritten Fortsatz; die andere liess nicht weniger als sechs Fortsätze erkennen. Eo war nicht zu entscheiden, ob alle zur Peripherie ziehenden Zellfortsätze zu Nervenfasern wurden, oder nicht. Bei einem anderen Exemplar wurde ein Fortsatz nicht zu einer Nerveufaser, sondern trat an eine benachbarte Ganglien- 
zelle heran und teilte sich nahe ihrer Oberfläche. Da die betreffende Zelle nur zur Hälfte geschwärzt war, im übrigen glücklicherweise zufällig einen bräunlichen Ton zeigte, so war es möglich, die Verästelung dieses Zellfortsatzes zu erkennen, und Disse hielt diesen Fortsatz für einen Dendriten. Es musste dahingestellt bleiben, ob einige der kurzen Zellfortsätze nicht auch in Wirklichkeit Fortsätze benachbarter Zellen waren, die an die Zelle herantraten und sich auf ihr verästelten.

Weiter schreibt Disse noch Folgendes : „Nun wird allgemein angegeben, dass in den Spinalganglien erwachsener Frösche nur unipolare Zellen vorkommen. Weun das richtig ist-was ich zur Zeit nicht bestreiten kann-so bleibt nur übrig anzunehmen, dass eine Auzahl von Zellausläufern während des Wachstums wieder schwindet. Dieser Vorgang bedarf der Erforschung; es muss nachgewiesen werden, ob ein Schwinden von Zellfortsätzen wirklich an Spinalganglienzellen vorkommt, oder ob sich diese Fortsätze erhalten und nur bisher übersehen worden sind. Beides ist möglich, und ich hoffe, durch weitere Untersuchungen die Frage entscheiden zu können. Jedenfalls ist eine Aenderung des morphologischen Verhaltens von Nervenzellen, bestehe sie nun in einer Vermehrung oder in einer Rückbildung der Zellfortsätze, in einer Vervielfältigung oder einer Vereinfachung der Verbindungen, die die Zelle besitzt, für das Verständnis der Leistungen dieser Zellen von der höchsten Wichtigkeit und muss darum genau untersucht werden."

In der Diskussion bemerkte v. Lenhossék(2) an Disses Präparaten das Vorkommen von multipolaren Zellen in den Spinalganglien der Froschlarven und bedauerte nur, dass die Präparate keine weitere Verfolgung der Ausläufer gestatteten. Immerhin aber schienen ihm alle Fortsätze den Charakter von Nervenfortsätzen zur Schau zu tragen, und er erklärte sich entschieden gegen die Auffassung cer überzähligen Ausläufer als Dendriten. Die richtige Erklärung für Disses Befund schien ihm die zu sein, dass bei Froschlarven vielleicht wegen der Grösse und der gedrängten Lagerung der Elemente an manchen Spinalganglienzellen die ersten Teilungen der beiden Acheenzylinder auf die Zelle selbst gerückt sind, wodurch eine vorübergehende Vermehrung der Ausläuferzahl veranlasst wird. Jedenfalls handelt es sich nach ihm bloss um eine embryona'e Erscheinung.

Im folgenden Jahre liess v. Lenhossék (13) die Bederken, die er in 
Göttingen Disses Deutung gegenüber ausgesprochen hatte, fallen und schloss sich nun Disse darin an, dass er die überzähligen Ausläufer auch beim Frosch für Dendriten hielt. Er fand nämlich, dass auch beim Hühnchen in einem vorgerückteren Stadium der Embryonalentwicklung sporadisch Zellen in den Spinalganglien auftreten können, bei denen sich neben ihren beiden typischen Fortsätzen, dem zentralen und dem peripherischen, noch eine Anzahl kurzer, teilweise verzweigter, entschieden als Dendriten aufzufassender Ausläufer entwickeln. Der Ausdruck Dendriten war allerdings insofern nicht ganz passend, als von einer dendritischen Aufsplitterung keine Rede sein konnte. Die meisten Äste waren ungeteilt, und wenn auch eine Teilung vorlag, so handelte es sich stets nur um eine einfache Gabelung. Auf Grund dieser Tatsache meinte er, dass die gegenwärtigen Anschauungen über den Bau der Spinalganglienzellen der Vögel eine kleine Korrektur erfahren müssten. Weiter untersuchte er mit der Golgischen Methode nicht nur bei neugeborenen, sondern auch bei einige Tage alten Mäusen -bis zum 8. Tage-die Nervenzellen sowohl in den Spinalganglien als auch in den Ganglien der Hirnnerven, wobei er nur unipolare Formen nachweisen konnte. Bei Säugern scheint also nach ihm die bei Hühnchen und Frosch nachgewiesene Erscheinung nicht vorzukommen. Auch van Gehuchten, der die Spinal- und Kopfganglien von Säugern untersucht hat, scheint nur unipolare Formen gefunden zu haben.

Ramón y Cajal(1) hat in den Spinalganglien von Hühnchen sporadische Elemente angetroffen, von welchen neben den typischen peripheren und centralen Nervenfortsätzen auch einige kurze und nicht selten sich verzweigende dendritenförmige Fortsätze abgingen. Bezüglich der Bedeutung dieser multipolaren Zellen glaubt R. y Cajal, dass ihre kurzen Dendriten aller Wahrscheinlichkeit nach in Folge einer regressiven Metamorphose zu Grunde gehen.

Im Jahre 1894 erwähnte Retzius $(18,19)$ multipolare Zellen in den Spinalganglien eines $2 \mathrm{~cm}$ langen Fötus von Mus decumanus und von Ophidien. Bekanntlich hat zuerst R. y Cajal, und im Anschluss an ihm van Gehuchten, die Ansicht ausgesprochen, dass die peripherischen(sensiblen) Fortsätze der Spinalganglien als Dendritenfortsätze, die zentralen dagegen als Axenzylinder aufzufassen seien. Dieser Auschauungsweise soll Retzius angeblich schon längst beigetreten sein. 
Kölliger(11) bringt in seinem Lehrbuch der Histologie die Zeichnung einer multipolaren Zelle aus dem Ganglion Gasseri vom Kalbe.

Spirlas(22) hat eine Untersuchung der Spinalganglien der Säugetiere mittelst der Golgi schen Methode vorgenommen, aus deren Resultaten hervorgeht, dass auch die Spinalganglienzellen der Säugetiere, wenigstens in der Embryonalzeit, eine Anzahl von multipolaren Zellen in sich einschliessen. Diese Beobachtung gelang ihm besonders schön an dem Embryo einer Ziege von $9 \mathrm{~cm}$ Länge. An diesem Embryo befanden sich die meisten Zellen der Spinalganglien noch im Stadium der Bipolarität. Einige zeigten ihre beiden Fortsätzo nahe an einander gerückt, und sehr wenige waren unipolar geworden. Unter den bipolaren Zellen gab es mehrere Exemplare; die ausser dem centralen und dem peripheren Fortsatze noch eine Anzahl anderer Auslänfer entsendeten. Diese sekundären Ausläufer erschienen entweder unverzweigt, oder sie teilten sich hier und da in zwei oder drei Aestehen, deren weiteres Verhalten Spirlas' Präparaten nicht zu ermitteln war. Die genannten Ausläufer, die er als Dendriten betrachtete, entsprangen hãufiger am peripheren Pol der Zelle. An manchen Zellen entsprang der periphere Fortsatz gemeinschaftlich mit dem Dendriten, wobei es den Anschein hatte, als ob der Dendrit ein Seitenästchen des peripheren Fortsatzes wäre. Spirlas sah an seinen Präparatén häufig kleine Seitenästchen von beiden Hauptausläufern, besonders aber vom peripheren, ausgehen. Leider war ihr Verlauf nur kurz, und Spirlas vermochte sie nicht weiter zu verfolgen. Er meint, dass es gewiss von Bedeutung wäre, wenn die genannten Aestchen sich.also konstant und verästelt exwiesen, da sie dann offenbar dąu dienen würden, die Spinalganglienzellen unter eiuander in Beziehung zu bringen. Ferner konnte Spirlas ganz deutlich eine Teilung der beiden Hauptausläufer in zwei gleichwertige Aeste beobachten, so dass im von ihm abgebildeten Falle eine Zelle durch die 'Teilung des zentralen Ausläufers mit drei Nervenfasern versehen war. Nach seinen Beobachtungen werden díe genannten Zellen gleich den übrigen unipolar, mit dem Unterschied jedoch, dass sich der Stammfortsatz derselben statt in zwei in drei Nervenfasern teilt.

Dogiel (4) hat die Spinalganglien bei erwachsenen Säugetieren (Hunde, Katzen, Kaninchen und Meerschweinchen) mittels der von ihm modifizierten Methode mit Methylenblau untersucht. Die Untersuchung erstreckte sich anf alle Spinalganglien und das Ganglion Gasseri. Er schreibt daüber Folgendes : 
„, Meine Beobachtungen zeigen nun; dass die multipolaren Zellen nicht nur in den Spinalganglien von Embryonen, sondern auch bei erwachsenen Tieren vorkommen. Sie sind von unregelmäsig eckiger Form und ziemlich bedeutender Grösse. Von den Ecken einer jeder solchen Zelle gehen mehrere $(4,10,12)$ glatte oder variköse Fortsätze von verschiedener Dicke aus, welche die Zellkapsel durchbohren und sich dann zwischen den unipolaren Zellen des entsprechenden Ganglions verzweigen. Ihrem Aussehen nach gleichen diese Zellen sehr den sympathischen und färben sich ziemlich schwer mit Methylenblau. Die Zahl der multipolaren Zellen mag in jedem Ganglion eine sehr beschränkte sein, da ich auf meinen Präparaten in einigen Ganglien nur wenige (1, 2 bis 3) sah ; in anderen schienen aber gar keine zu sein; wenigstens wurden durch Methylenblau keine gefärbt, obgleich die Färbung vieler unipolaren Zellen intensiv und vollständig war. Hiernach kann es also nicht mehr zweifelhaft sein, dass die multipolaren Zellen nicht nur im Embryonalleben der Tiere existieren, sondern ein ständiges. Element der Spinalganglien bilden und zweifellos eine gewisse physiologische Bedeutung besitzen."

Auch Huber (10) fand bei Chelhydra und Hühnchen multipolare Zellen, über die er Folgendes schreibt: „In the dorsal spinal ganglia of Chelhydra serpentina it is not unusual to find a small group of multipolar cells within the capstule of the ganglion. They are found on the ventral sicle of the ganglion and are surrounded by pericellular baskets. The axis cylinders of these cells are very fine and varicosed. The cells, their axis cylinders and dendrits, as also the pericellular baskets enclosing them, have every appearance of sympathetic cells. I am inclined to think that they are such in Chelhydra. - My observation on the multipolar cells in the spinal ganglia of the chicken lead me to similar conclusions. As far as I have been able to determine, I am inclined to believe that some few sympathetic cells are found within the capsule of their dorsal spinal ganglia, a larger number being found extra-capsular. The cells observed by me possess only one axis cylinder process, differing in this respect from the cells described by Lenhossék."

Van Gehuchten(8) schrieb: ", Nous avons reproduit dans la fig. 2 quelques types de cellules multipolaires que nous avons observés dans les ganglions spinaux d'embryons de Tropidonotus de 10 centimetres de longueur. Quelle est. la signification de ces cellules multipolaires ?" dann erwähnte er Fälle von Disse, 
v. Lenhossék und Retzius und fuhr fort: "Les prolongements surnuméraires sont pour nous des prolongements protoplasınatiques; nous partageons en cela l'opinion de Disse et de Lenhosoék."

Dogiel (6) hat bei Säugetieren und bei Menschen solche multipolare Zellen in den Spinalganglien gefunden und sagt : „Die hier angeführten Tatsachen weisen darauf hin, dass einige, vielleicht auch alle Spinalganglien eigentlich einen gemischten Charakter haben: die Mehrzahl ihrer Zellen gehört zu den Spinalganglienzellen der verschiedenen Typen, doch finden sich unter ihnen augenscheinlich auch sympathische Zellen in beschränkter Anzahl.“

Retzius(20) erkannte bei Embryonen von Vespertilio auritus ron 12,5 mm Länge in einem Ganglion eine verästelte Zelle, indem vom Zellenkörper selbst ein kleiner, verzweigter Fortsatz auslief; von den beiden Enden der Zelle ging je eine Faser ab, die jedoch nicht weiter zu verfolgen war. In Betreff der Bedeutung stimmt Retzius van Gehuchten darin bei, dass es sich bei diesen Fortsätzen um keine spezifischen, fest differentierten handele, sondern dass sie mehr als eine Aberration, eiu Anklang an die Gestalt der Rückenınarkszellen, aufzufassen, also als eine Entwicklungoform zu betrachten seien, die sich wahrscheinlich zurückbilde, indem sie nämlich in den embryonalen Ganglien nur ganz vereinzelt und ohne bestimmte Lagerung vorkommen. In den zwei von ihm genau durchmusterten Kaninchenembryonen, wo die Zellen der Spinalganglien zahlreich und gut gefärbt waren, traf er im ganzen kaum zehn solche verästelte Zellen in gefärbtem Zustande an.

Retzius (21) soll auch bei den Ophidiern und bei einen 5 tägigen Hühnerembryo in dem Spinalganglion die Multipolarzellen gefunden haben.

Es soll Holmgren(9) sehr oft durch Färbung mit Eisenhämatoxylin gelungen sein, in den Spinalganglien des erwachsenen Lophius Zellen mit dendritischen Fortsätzen nachzuweisen und diese letzteren ziemlich weit von den bezüglichen Zellen zu verfolgen. Er hat 3-8 solche von den Zellen auslaufende Fortsätze angetroffen. Die Tigroidelemente des Zelleibes setzten sich in diese fort. Bald ganz in der Nähe des Zellkörpers, bald mehr oder weniger davon entfernt teilten sie sich gabelförmig.-Um ihr peripherisches Schicksal näher verfolgen zu können, wären indessen andere Methoden als die von ihm benutzten notwendig gewesen, und er musste sich deshalb darauf beschränken, nur das Vorhandensoin multipolarer Zellen in den Spinalganglien von Lophins hervorzu- 
heben.-Er will nicht behaupten, dass dendritische Fortsätze von allen Spinalganglienzellen ausgehen; demn er fand umgekehrt auch Zellen, an denen solche Fortsätze ganz vermisst wurden. Jedenfalls aber glaubt er behaupten zu dürfen, lass multipolare Zellen sehr allgemein sind.

Levi(15) beschreibt in den Spiualganglien der Chelonier multipolare Zellen mit kurzen und dicken Fortsïtzen, welche nicht selten in sehr grossen kenlenförnigen Anschwellungen endigen. Er hält diese Fortsätze für echte Dendriten. Bei den Selachiern sind nach den Beobachtungen von Levi(16) in den Spinalganglien ebenfalls Zellen vorhanden, von denen an verschiedenen Stellen häufig recht dicke Fortsätze abgehen. Sie treten unter der Kapsel hervor, verzweigen sich in einigen Fïllen und endigen daranf in Anschwellungen.

Im Jahre 1906 gab R. y Cajal (2) die wichtigsten Resultate ssiner Untersuchungen, die schon in spanischer Sprache veröffentlicht worden, durch Abbildungen erläntert wieder. Dannch schien das Vorhandensein der aus dem Zellkörper stammenden dendritischen Ausbreitungen am erwachsenen Menschen mit Hilfe der Silberreduktion vollkommen nachreisbar zu sein. Weiter schreibt er : "Nichts!estoweniger ist es sehr schwer zu bestimmen, ob die Zellen, welche wir bein erwachsenen Menschen und vorzüglich beim Greis gefunden haben, in Wïklichkeit den von Disse, v. Lenhossék, Spirlas und Dogiel bei verschiedenen Vertebraten beschriebenen entsprechen; denn man darf nicht vergessen, dass die Dendriten der dem Menschen zugehörenden Elemente dick und verästelt sind und im Innern der Kapsel mit einer mehr oder weniger voluminösen Verlängerung endigen, während die Dendriten, welche aus den bei dem Frosch, den Vogelembryonen, der Katze etc. beschriebenen Zellen hervorgehen, einen viel kleineren Durchmesser besitzen und ausserhalb der Kapsel mit nicht verdickten Endigungen abschliessen. Doch lassen wir für den Moment die Frage der Identification dieser beiden Arten ganglionärer Zellen beiseite und beschränken uns auf die Auordnungen, wie sie durch die Silberreduktion beim Menschen und den grosen Säugetieren (Esel, Pferd, Rind etc.) aufgefunden sind, so bemerken wir, dass nan bei diesen Tieren zwei Arten mit Dendriten versehener Körperchen unterscheiden kann, die kleine und die voluminüse Varietït."

Nageotte (17) fand bei der Untersuchung transplautierter Spinalganglien des Kaninchens in denselben multipolare Zellen. Darüber schreibt er folgender- 
massen : „mais il existe de nombreux prolongements, les uns très fins, les antres plus gros, qui partent de $h$ cellule et rayonnent dans tous les sens. Les prolongements les plus fins naissent soit directement du corps cellulaire, soit des prolongements plus épais au voisinage de la cellule; ils entrent pour la plupart dans la formation, d'un plexus sous-capsulaire complique qui enserre le corps cellulaire ; d'autres s'etendent au loin. Les prolongements les plus volumineux, au nombre de trois ou quatre au moins, affectent une disposition singulière ; à peu de distance de la cellule, ils presentent une série de renflements irréguliere; souvent très volmineux, qui donnent naissance à un grand nombre de branches; parmi ces branches, les unes sont courtes et trapues, terminées par des boules; les autres sont plus minces, longues, renflées à leur tour et ramifiées à l'infiui ; les ramifications ultimes c'e cette arborisation difforme sont constituées par d'innombrables fibres très fines, terminées par des boubles, qui forment des bouquets au voisinage 'ie la cellule et qui rappellent par leur disposition certaines terminaisons nerveuses sensitives."

$v$. Lenhossék (14) hat die multipolaren Zellen in den Spinalganglien des Menschen wiedergefunden, allerdings auch hier nur als sporadische Elemente. Er schreibt: „Die Beobachtungen aber, die ich über das Verhalten dieser Fortsätze machen konnte, weichen von der bisherigen Auffassung sehr betrïchtlich ab und lassen sie in cinem ganz anderen Lichte erscheinen. Ich finde vor allem, dass diese Fortsätze, von denen man an einem Durchschnitt der Zelle oft sechs, sieben nachweisen kann, durchaus nicht den Charakter von Dendriten haben. Sie stellen sich vielmehr vorkommen in der Glätte und Schärfe echter Nervenfortsätze c'ar und sind von sehr verschiedener Dicke. Manchmal sind es grobe Balken, viel häufiger aber zarte Fäserchen, die mit einem kleinen hügelartigen Anfangsstück ziemlich unvermittelt von der Oberfläche der Zelle entspringen. Was aber am meisten überrascht, ist das weitere Verhalten dieser Fortsätze. Geht man ihnen nämlich nach, so findet man, vorausgesetzt, dass sie nicht gleich an ihrem Ursprunge oder mach kurzem Verlauf abgeschnitten sind, dass sie innerhalb der Kapsel zwischen den Mantelzellen in bogenförmigen und winkligen Anastomosen mit Nachbarfortsätzen ihr Ende finden, und zwar bilden nicht nur je zwei mit einander solche Verbindungen wie es bei den oben beschriebenen Schlingen der Fạll ist, sondern es können sich mehrere solche Fortsätze nach einander gegenseitig vereinigen, so dass um die Nervenzelle herum 
ein zierliches, von der Zelloberfläche weit abstehendes weitmaschiges Gitterwerk zustande kommt."

Schliesslich schreibt Dogiel(7) wieder über diese Zellen. Nach ihm sollen die multipolaren Zellen unter den übrigen Zellformen eine Sonderstellung einnehmen. Es handelt sich seiner Meinung nach um junge, im Wachstum begriffene Elemente, welche als Eroatz für alternde bezw. absterbende Zellen der verschiedenen beständigen Typen dienen.

Nach der oben chronologisch angegebenen Literatur wurden die Multipolarzellen in den Spinalganglien zuerst von Disse bei Froschlarven beschrieben. Darauf wurden sie von mehreren Autoren bei verschiedenen, auch erwachsenen Tieren gefunden. Vom Rinde hat Kölliker in seinem Lehrbuch eine solche Zelle aus dem Ganglion Gasseri eines Kalbes abgebildet.

Auch mir ist es gelungen, mittels der Cajalschen Methode die multipolaren Zellen im Ganglion Gasseri eines erwachsenen Rindes aufzufinden. Was die Untersuchungsmethoden anbetrifft, so erlaubt die vitale Methylenblaufärbung die Anfertigung verhältnismässig dicker Schnittpräparate wodurch man das weitere Schicksal einer Faser leicht verfolgen kann. Hinsichtlich der Schärfe übertrifft jedoch diese Methode nicht die Cajalsche, welch letztere in manchen Beziehungen für ein eindringlicheres Studium vorzuziehen ist. Ich will hier kurz die von mir benutzte Methode beschreiben.

Ein kleines Stück des Ganglion semilunare des Nervus trigeminus wird 24 Stunden lang in absolutem Alkohol mit Zusatz von 0,5\%igem Ammoniak gehärtet. Darauf wird es flüchtig in destilliertem Wasser abgewaschen und in eine 2\% ige Silbernitratlösung gebracht und 3-5 Tage lang bei $36^{\circ} \mathrm{C}$. darin gelassen. Nach ganz kurzem Abspülen in dest. Wasser kommt das Stück 24 Stunden lang in eine Mischung aus Pyrogallussäure 2,0, Formalin 5,0 und Aq. dest. 95,0, wird dann wieder einige Minuten mit dest. Wasser gewaschen durch Alkohol entwässert und schliesslich in Paraffin eingebettet. Das Stück wird darauf in Schnitte von der verschiedenen Dicke von 25-30-10 zerlegt. Einen Teil der Schnittpräparate habe ich nach v. Lenhossék vergoldet.

Da die Schnittpräparate je nsch dem Grade der Imprägnation vom,Centrum nach der Peripherie verschiedene Nuancen, gelb, braun, schwarz darbieten, so kann man sich leicht eine geeignete Stelle zur Untersuchung aussuchen. Zur Untersuchung benutzte ich ausser dem gewöhnlicher Mikroskop: noch Zeiss' 
binokularen Tubenaufsatz „Bitumi“. Bei einer grossen Zelle wie die Ganglionzelle, deren Fortsätze einen sehr verwickelten Verlauf aufweisen, scheint mir die stereoskopische Beobachtung die zweckmässigste zu sein. Ausser echt stereoskopischer Beobachtung gestattet der Apparat „Bitumi “ durch Drehung der Okulardeckel die Wiedergabe eines pseudostereoskopischen Bildes, in dem die Erhabenheiten sich vertiefen und die Vertiefungen sich erheben. Nach meiner eigenen Erfahrung ist dieser Apparat für die hier in Frage kommenden Untersuchung ein unentbehrliches Hilfsmittel.

Im Ganglion semilunare des ausgewachsenen Rindes finden sich ausser den gewöhnlichen Unipolarzellen und verhältnismässig selten anzutreffenden Bipolarzellen noch zerstreut multipolare Zellen vor. Was die Häufigkeit der einzelnen

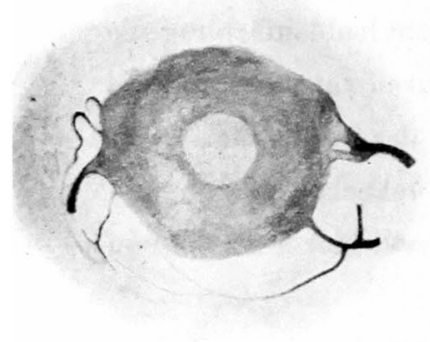

a

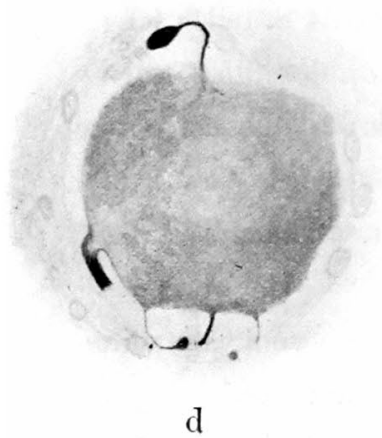

d

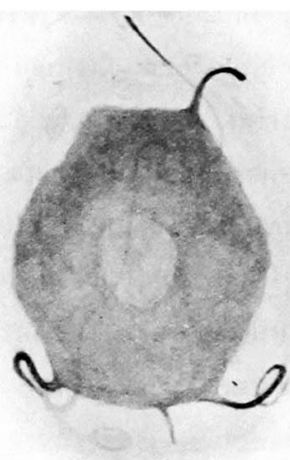

$\mathrm{b}$
Finige Multipolarzellen aus dem Ganglion senilunare des Rindes. 470 mal vergrössert. 
Zellarten anbetrifft, so zählte ich bei meiner Durchmusterung von 50 Gesichtsfelclern 2801 Unipolar- : 55 Bipolar- : 12 Multipolarzellen.

Die Multipolarzellen sind mittelgross, rundlich oder oval und mehr oder weniger polygonal. Das Zytoplasma ist im allgemeinen gelblich oder bräunlich gefärbt und körnig. Der kuglige Kern ist blasser gefärbt und liegt in der Mitte der Zelle. Wie auf den beigelegten Figuren abgebildet, entspringen aus der Oberfläche des Zelleibes 3-6 Fortsätze, deren Anfangsabschnitte sich mehr oder weniger kegelförnig erheben und allmählich verjüngen. Der Anfangskegel ist mehr oder weniger blass gefärbt und die Ausläufer dagegen im weiteren Verlauf dunkler geschwärzt, so dass man sie dadurch ohne weiteres vom Zelleib leicht unterscheiden kann. Doch gibt es auch ein paar feine Fädchen, die denselben Farbenton wie der Zelleib haben. Die Fortsätze sind inbezug auf Grösse sehr verschieden. Dendritische Verzweigungen trifft man sehr selten an, und ihre Enden verschwinden in cler Zellkapsel (Fig. a, links, unten). Die abgeschnittenen Enden der Fortsätze enden entweder inner- oder ausserhalb der Zellkapsel, nachdem die Fortsätze nach etwelchen Windungen aus der Zellkapsel herausgetreten sind. Über den Charakter der Fortsätze kann man nichts mit Sicherheit aussagen, weil sich hier ihr Verlauf nicht weiter verfolgen lässt. Doch scheinen mir einige durch ihre Dicke und ihren Verlauf als Nervenfortsätze (Fig. c) und andere als Dendriten (Fig. a, d) in Betracht zu kommen, indem diese letzteren wegen ihrer Feinheit nicht als Nervenfasern angesprochen werden können, obgleich die dendritischen Verzweigungen fehlen. Besonders kann man feinere Fälchen mit ganz dem gleichen Farbenton wie dem des Zelleibes ohne weiteres als Dendriten auffassen (Fig. b, d, unten). Der Anfangsabschnitt eines Hauptfortsatzes erscheint besonders blass gefärbt, was auch bei den Unipolarzellen der Fall ist. Dies kommt dadurch zustande, dass sich in der Nähe der kegelförmigen Anfangserhabenheit der Zelleib in ein Grübchen einzieht. Bei einem Exemplar tritt ein Nervenfortsatz mit zwei Wurzeln zutage, die sofort nach ihrem Entspringen aus dem Zelleib wieder zusammenfliessen (Fig. a). Ob in der Weise zwei Fortsätze schliesslich zu einen Fortsatz wercen, es sich also um eine Ubergangsform handelt, will ich vorläufig dahingestellt sein lassen. Bei einem anderen Exemplare treten ausser einem ansehnlichen Hauptfortsatze noch ein paar feine Fädchen hervor, die mit kugeligen oder keulenförmigen Anschwellungen inner- oder unterhalb der Kapsel enden und keine Verästelung aufweisen 
(Fig. d). Die Kerne der Zellen sind nach unserer Methode weder in der Kapsel noch in der Nervenscheide deutlich zu sehen. Nach v. Lenhossék soll dabei zur Darstellung mit bestem Erfolg das May er sche Karmalaun angewandt werden.

Wie wir oben gesehen haben, kommen auch bei dem erwachsenen Rinde die multipolaren Zellen im Ganglion semilunare zerstreut vor. Betreff des Charakters ihrer Fortsätze sind diese meiner Ansicht nach teils als Dendriten, teils als Nervenfortsätze aufzufassen.

Zum Schluese sei mir gestattet, Herrn Prof. Dr. S. F unaoka für seine freundliche, stete Unterstützung meinen verbindlichsten Dank auszusprechen.

Kyoto, Oktober 1924. 


\section{Literatur.}

1. Cajal, Ramón $y$, Los ganglios $y$ plexos nerviosos del intestino de los mamiferos $\mathbf{s}$ pequenas adiciones a nuestros trabojos sorbe la médula $y$ gran sinpáties general, Madrid 1893, p. 44. (Zitiert nach Dogiel 1897).

2. Derseibe, Die Struktur der sensibler Ganglien deg Menschen und der Tiere. Ergebnisse der Anat. u. Entw. Bd. 16. 1906. S. 177.

3. Disse, Über die Spinalganglien der Amphibien. Anat. Anz. Suppl. 2. VIII. 1893, Verhandl. d. Anat. Gesellsch. auf d. VII. Vers. 1893, S. 201.

4. Dogiel, Der Bau der Spinalganglien bei den Säugetieren. Anat. Anz. Bd. 12. 1896, S. 140.

5. Derselbe, Zur Frage über den feineren Bau der Spinalganglien usw. Journal intern. d'anatomie, $1 \leqslant 9 \%$.

6. Derselbe, Zur Frage über den Bau der Spinalganglien beim Menschen und bei den Säugetieren. Intern. Monatsch. f. Anat. u. Physiol. 1898, S. 345.

7. Derselbe, Der Bau der Spinalganglien des Menschen und der Säugetiere. Jena, 1908.

8. van Gehuchten, Contribution a l'etude de la moelle epiniere chez les vertebres. La Cellule 1897, T. 12. fase. 1. p. 122.

9. Holmgren, Zur Kenntnis der Spinalganglien von Lophius picatorius Lin. Anat. Hefte I Abt., H. 38. Bd. 12. 1899.

10. Huber, The Spinal Ganglia of Amphibia. Anat. Anz. Bd. XII. No. 18. 1896.

11. Kölliker, Handb. d. Gewebelehre d. Menschen. Bd. 2, 1896.

12. Lenhossék, Discussion. Verhandl. d. Anat. Gesellsch. auf d. VII. Vers. 1893, ‥ 204 .

13. Derselbe, Beiträge der Histologie des Nervensystems. Wiesbaden 1994, S. 12i.

14. Derselbe, Zur Kenntnis der Spinalganglienzellen. Arch. f. mikr. Anat. u. Entw. Bd. 69. 190־, S. 245.

15. Levi, La struttura dei gangli cerebrospinali dei cheloni. Monitore Zoologico Italiano. Anno 17. No. 4. 1906 (Zitiert bei Jahresber. d. Anat. X. F. Bd. 12. 1903, 1. Teil, S. 298).

16. Derselbe, La str. d. gangl. cerebro-sp. nei Selaci e nei Teleostei. Monit. Zoolog. Italiano. Anno 17. No. 8. 1906. (Zitiert nach Dogiel 1908).

17. Nageotte, Greffe de ganglions rachidiens, survie des éléments nobles et transformation des cellules unipolaires en cellules multipolaires. Comptes rendus des séances de la Société de Biologie. T. LXII, 1907, p. 62. 
18. Retzius, Zur Entwicklung der Zellen des Ganglion spirale acustici und zur Endigungsweise des Gehörnerven bei den Säugetieren. Biolog. Untersuchungen T. VI. 1894, S. 52.

19. Derselbe, Die embryonale Entwicklung der Rückenmarkselemente bei den Ophidiern. Biolog. Unters. T. VI. 1894, S. 41.

20. Derselbe, Zur Kenntnis der ersten Entwicklung der Rückenmarkselemente bei den Säugetieren. Biolog. Unters. T. VIII. 1898, S. 102.

21. Derselbe, Weiteres über die embryonale Entwicklung.der Rückenmarkselemente der Ophidier. Biolog. Unters. T. VIII. 1898, S. 105.

22. Spirlas, Zur Kenntnis der Spinalganglien der Säugetiere. Anat. Anz. Bd. 11. 1896, S. 629. 